\title{
Low-Intensity Shockwave Therapy in the Treatment of Erectile Dysfunction
}

Kelly Lurz ${ }^{1}$, Paulette Dreher ${ }^{2}$, Jason Levy ${ }^{2}$, Brian McGreen ${ }^{2}$, Javier Piraino ${ }^{2}$, Andrew Brevik ${ }^{3}$, Daniel Edwards ${ }^{2}$, Laurence H. Belkoff 4

1. Urological Surgery, Mainline Health, Philadelphia, USA 2. Urology, Mainline Health, Philadelphia, USA 3. Urology, Kansas City University of Medicine and Biosciences, Kansas City, USA 4. Surgery, Mainline Health, Philadelphia, USA

Corresponding author: Kelly Lurz, lurzkelly@gmail.com

\section{Abstract}

\section{Introduction}

Low-intensity shockwave therapy (LISWT) may improve erectile function in patients with mild to moderate erectile dysfunction (ED). Currently there is a paucity of research and prospective data on the utilization of LISWT in patients with ED. We present the results of our phase II clinical trial of LISWT with short-term follow-up in a cohort of patients with mild to moderate vasculogenic ED.

\section{Methods}

We obtained IRB approval and prospectively enrolled patients with mild to moderate vasculogenic ED. Baseline International Index of Erectile Function (IIEF) scores and peak systolic velocities (PSV) of cavernosal arteries measured on duplex penile ultrasound were obtained prior to treatment. Treatment included 6600 total shocks per session, for a total of six consecutive weekly treatment sessions. Baseline Erectile Dysfunction Inventory of Treatment Satisfaction (EDITS) scores were obtained at the completion of the treatment course. IIEF, EDITS and PSV were evaluated again at one-month follow-up. Clinical significance was defined as a median IIEF score increase of four points from baseline or an EDITS total score increase to greater than 65 or increase of greater than ten from baseline. Treatment success was evaluated on an individual basis and defined by a clinically significant improvement in questionnaire score.

\section{Results}

A total of 25 patients were enrolled in the trial, with 22 patients reporting for one-month follow-up. $68 \%$ $(15 / 22)$ of patients demonstrated treatment success. In the cohort there was improvement in median EDITS from 61 (IQR 49-92) to 73 (IQR 43-49), which did meet criteria for clinical significance, but did not reach statistical significance $(\mathrm{p}=0.74)$. IIEF improved from a median of 13 (IQR 12-19) to 18 (IQR 14-25), which did reach statistical significance $(\mathrm{p}=0.011)$. On duplex ultrasound, mean cavernosal artery PSV increased from $34.3 \mathrm{~cm} / \mathrm{s}$ (IQR 25.7-51.1) to $38.0 \mathrm{~cm} / \mathrm{s}$ (IQR 31.6-45.1); however, these differences were statistically insignificant $(\mathrm{p}=0.986)$. Of the 25 patients undergoing LISWT, two reported discomfort during treatment sessions, which subsided after repositioning the device without alterations in energy delivered.

Review began 09/15/2020 Review ended 10/23/2020 Published 11/01/2020

\section{() Copyright 2020}

Lurz et al. This is an open access article distributed under the terms of the Creative Commons Attribution License CC-BY 4.0., which permits unrestricted use, distribution, and reproduction in any medium, provided the original author and source are credited.

\section{Conclusion}

LISWT may be a safe and potentially efficacious clinical modality for treatment of patients with mild to moderate vasculogenic ED demonstrating increases in cavernosal artery PSV and improvements in IIEF and EDITS scores in short-term follow-up. Longitudinal studies with increased power are needed to better evaluate the long-term efficacy and cost-efficiency of this therapy.

Categories: Urology

Keywords: erectile dysfunction, extracorporeal shockwave therapy, surveys and questionnaires

\section{Introduction}

Erectile dysfunction (ED), described by the American Urological Association (AUA) as the inability to achieve and maintain an erection sufficient for satisfactory sexual intercourse, is estimated to affect upwards of 30 million men in the United States. Arising from both organic and psychogenic etiologies, over 600,000 American men are diagnosed with ED every year [1]. Of all organic ED etiologies, vasculogenic causes are the most extensive in the United States, likely related to the prevalence of obesity, cardiovascular disease, and diabetes in Western society [2]. The management of vasculogenic ED has historically been focused on treatment of the symptom, flaccidity, rather than long-term correction of the underlying mechanism attributed to vascular and endothelial dysfunction. However, studies have demonstrated that lifestyle modifications, such as diet, exercise, weight loss, and smoking cessation may contribute to long-term improvements in ED, likely due to improved endothelial function [3]. 
The clinical diagnosis of ED is most commonly made with the assistance of validated questionnaires; the International Index of Erectile Function (IIEF) and Erectile Dysfunction Inventory of Treatment Satisfaction (EDITS) are two of the most frequently used. Along with identifying the nature of ED, these questionnaires can be utilized to monitor symptom improvement and treatment outcomes. While oral phosphodiesterase5-inhibitors (PDE5-Is), vacuum devices, intraurethral alprostadil, intracavernosal injections, and penile prosthesis surgery remain at the forefront of treatment for ED, research is surfacing regarding the implication of low-intensity shockwave therapy (LISWT) in the treatment of ED [4].

LISWT involves the application of linear shockwaves to target tissues, resulting in cellular microtrauma [4]. In turn, this microtrauma leads to the upregulation and release of angiogenic factors resulting in neovascularization of tissue [4]. LISWT has been utilized in many fields to stimulate tissue regrowth and regeneration, most notably in orthopedics for muscle, joint, and tendon recovery [5]. Clinical trials in human populations, although typically small and heterogeneous in population, have suggested improvements in erectile function after therapies employing LISWT to the corporal bodies [4,6-9].

The purpose of the current study was to evaluate the safety and short-term efficacy of LISWT in the treatment of mild to moderate ED and to consider potential factors associated with treatment success or failure in this population.

\section{Materials And Methods}

Internal Review Board (IRB) approval was obtained through the Western IRB to perform a non-randomized prospective phase II clinical trial.

\section{Patient selection}

Patients diagnosed with organic ED in a large urology group practice were screened for enrollment and referred to the central research clinic if baseline selection criteria were met. Research assistants then verified eligibility criteria and patients were enrolled in the study. Inclusion criteria included male patients between the ages of 35 to 75 years with a diagnosis of mild, mild to moderate, and moderate ED based on IIEF erectile function domain scores (22-25, 17-21, and 11-16, respectively), as well as a prior response to oral PDE-5I therapy, suggesting vasculogenic pathology and potentially reversible endothelial dysfunction [10]. Patients were limited to those reporting involvement in a heterosexual monogamous relationship with a minimum of three months duration and agreement from both partners to attempt intercourse a minimum of four times per month. Patients were excluded if they had a prior history of genitourinary malignancy, pelvic radiation therapy, implantable penile prosthesis, artificial urethral sphincter, uncorrected hypogonadism, substance abuse in the past three years or medical comorbidities including unstable angina, myocardial infarction in the last six months, congestive heart failure, or arrhythmias.

\section{Study design}

Subjective and objective outcomes were measured using pre- and post-treatment validated questionnaires and cavernosal artery velocities. Participants underwent a baseline screening evaluation including IIEF questionnaire and penile duplex ultrasound of the bilateral cavernosal arteries to determine peak systolic velocity (PSV). Patients then underwent treatment sessions once per week for six weeks using the RichardWolf PiezoWave low intensity shockwave lithotripter (Figure 1). Treatment sessions consisted of a total of 6,600 shocks (at $0.16 \mathrm{~mJ} / \mathrm{mm}^{2}$ ), with 3,300 shocks to each of the bilateral corpora via the penile shaft and proximally to the crura via the perineum. At completion of the six-week course, patients were evaluated with an EDITS questionnaire, which is referred to as "baseline" throughout this study. Patients underwent subsequent evaluation utilizing IIEF and EDITS questionnaires and PSV at one month following the conclusion of their treatment course. Dedicated research personnel administered questionnaires and a designated ultrasonographer performed LISWT treatments as well as penile duplex ultrasounds. PSV value for each patient was calculated as the arithmetic mean peak velocity of the two cavernosal arteries. 


\section{Cureus}

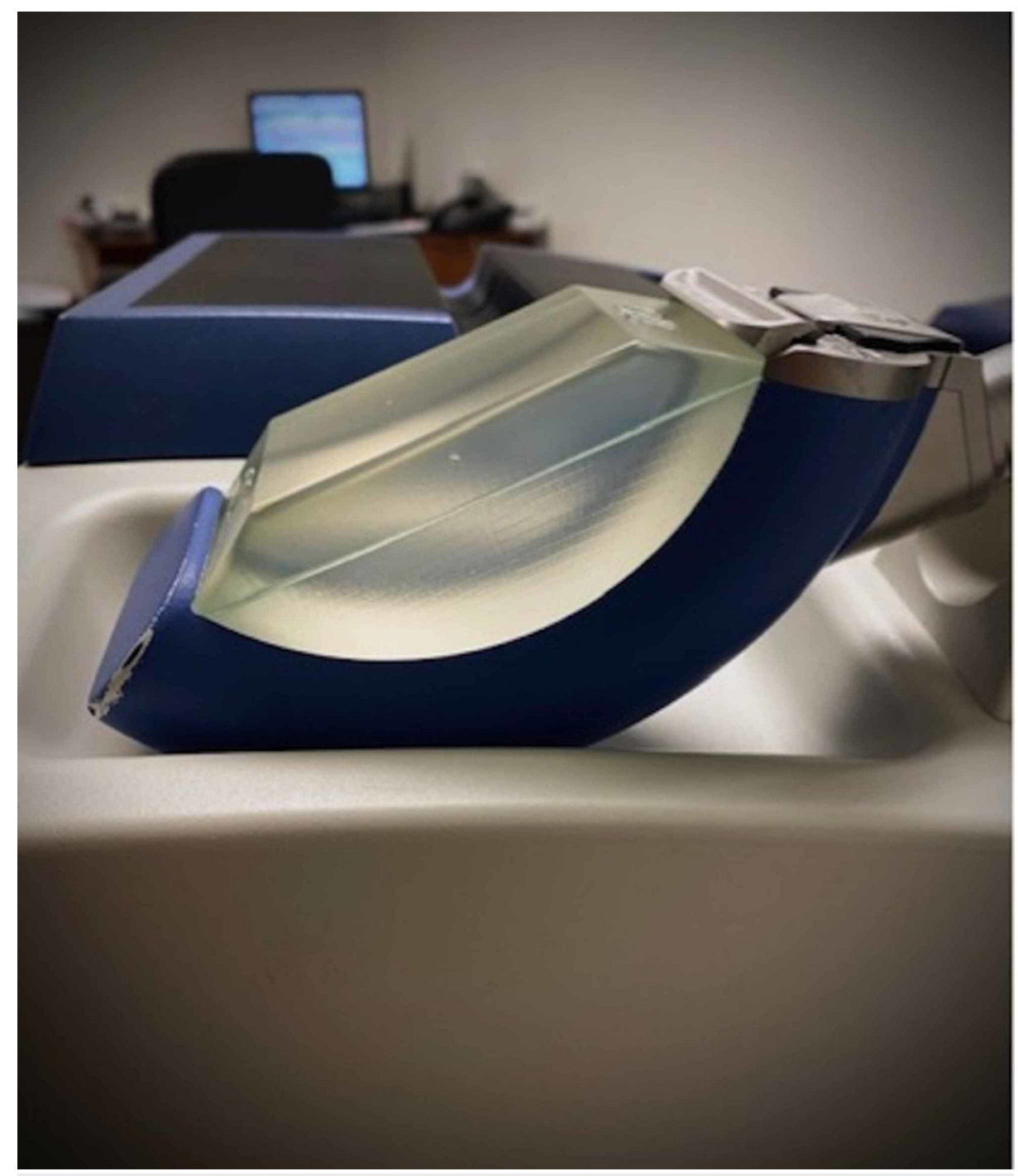

FIGURE 1: Richard-Wolf PiezoWave low intensity shockwave lithotripter

\section{Statistical analysis}

Statistical analysis was performed using IBM ${ }^{\circledR}$ SPSS ${ }^{\circledR}$ Statistics 24 (IBM Corp., Armonk, NY). Demographic and pre-treatment data were summarized by severity of baseline ED using medians and interquartile ranges for continuous variables and frequencies for categorical variables. Data were compared using Fisher's exact test for categorical variables and either Wilcoxon sign rank or Kruskal-Wallis ANOVA for continuous variables, as appropriate.

Clinical significance was defined as a median IIEF score increase of four points from baseline, or an EDITS total score increase to greater than 65 or increase of greater than 10 from baseline at one-month follow-up. Clinically significant changes in IIEF scores utilized in the evaluation of LISWT for ED vary across the literature; therefore we derived and utilized a modified average of these values [11-12]. Clinically significant values for changes in EDITS were derived from data published by Cappelleri et al. that described a clinically important difference on EDITS as a score increase of 10 points [13]. Treatment success was evaluated on an individual basis and defined by a clinically significant improvement in questionnaire score. Changes in PSV were evaluated for statistical significance only. Statistical significance for all metrics was defined at a $95 \%$ confidence interval with $\mathrm{p}$-value of $<0.05$.

\section{Results}

Twenty-five patients completed all six weekly treatment sessions and 22 patients had follow-up at one month. Demographics and pre-treatment data were categorized by severity of baseline ED. Patients had a median age of 66 years (IQR 55-69) for mild ED and 60 years (IQR 56-67) for moderate ED. Median duration of ED was reported as 8.5 years for mild and 4.0 years for moderate ED (Table 1). 


\section{Cureus}

\begin{tabular}{|c|c|c|c|c|c|}
\hline & & Mild $(n=4)$ & Mild to Moderate $(n=6)$ & Moderate $(n=15)$ & P-Value \\
\hline Age (years) & & $66(55-69)$ & $60(56-67)$ & $60(55-61)$ & 0.463 \\
\hline BMI (kg/m²) & & $27.5(24.9-33.8)$ & $32.7(24.4-37.6)$ & $30.5(27.9-33.5)$ & 0.655 \\
\hline Serum Testosterone (ng/dl) & & $333(223-633)$ & $404(256-824)$ & 571 (281-656) & 0.765 \\
\hline Duration of ED (years) & & $8.5(3.0-14.8)$ & $7.0(1.5-18.5)$ & $4.0(3.0-7.3)$ & 0.544 \\
\hline \multirow[t]{3}{*}{ Race } & & & & & 0.554 \\
\hline & African-American & 3 & 3 & 11 & \\
\hline & Caucasian & 1 & 3 & 4 & \\
\hline \multirow[t]{3}{*}{ Smoker } & & & & & 0.202 \\
\hline & Yes & 0 & 3 & 7 & \\
\hline & No & 4 & 3 & 8 & \\
\hline \multirow[t]{3}{*}{ Alcohol Use } & & & & & 0.736 \\
\hline & Yes & 3 & 5 & 10 & \\
\hline & No & 1 & 1 & 5 & \\
\hline \multirow[t]{3}{*}{ Hypertension } & & & & & 0.328 \\
\hline & Yes & 3 & 4 & 6 & \\
\hline & No & 1 & 2 & 9 & \\
\hline \multirow[t]{3}{*}{ Diabetes Melitus } & & & & & 0.482 \\
\hline & Yes & 0 & 1 & 4 & \\
\hline & No & 4 & 5 & 11 & \\
\hline
\end{tabular}

TABLE 1: Demographic data distribution by severity of baseline erectile dysfunction (ED).

On a per patient basis, $68 \%(15 / 22)$ experienced treatment success at one month post treatment. The calculated median EDITS score for all patients increased from 61 (IQR 49-92) to 73 (IQR 43-91) at one month, which is clinically, but not statistically significant $(\mathrm{p}=0.744)$. IIEF-6 scores, however, demonstrated both statistical and clinical improvements, increasing from a median of 13 (IQR 12-19) to 18 (IQR 14-25) at one month $(\mathrm{p}=0.011)$. Median EDITS and IIEF scores from baseline to one month follow-up for mild, mild to moderate, and moderate ED are displayed in Table 2 and Table 3, respectively. 


\section{Cureus}

\begin{tabular}{|c|c|c|c|}
\hline & Baseline & One Month & P-Value \\
\hline Overall & $61(49-92)$ & $73(43-91)$ & 0.744 \\
\hline Mild ED & $75(37-100)$ & $89(58-100)$ & 0.317 \\
\hline Mild to Moderate ED & $73(57-87)$ & $66(53-90)$ & 1.000 \\
\hline Moderate ED & $63(43-93)$ & $64(34-90)$ & 0.889 \\
\hline
\end{tabular}

TABLE 2: Changes in median EDITS scores from baseline to one-month post-treatment. Interquartile ranges listed in parentheses.

EDITS: Erectile Dysfunction Inventory of Treatment Satisfaction

\begin{tabular}{|c|c|c|c|}
\hline & Baseline & One Month & P-Value \\
\hline Overall & $13(12-19)$ & $18(14-25)$ & 0.011 \\
\hline Mild ED & $23(22-25)$ & $25(25-25)$ & 0.180 \\
\hline Mild to Moderate ED & $18(17-20)$ & $23(19-25)$ & 0.102 \\
\hline Moderate ED & $12(11-13)$ & $15(10-23)$ & 0.078 \\
\hline
\end{tabular}

TABLE 3: Changes in median IIEF scores from baseline to one-month post-treatment. Interquartile ranges listed in parentheses.

IIEF: International Index of Erectile Function

Cavernosal artery PSV increased from $34.3 \mathrm{~cm} / \mathrm{s}$ (IQR 25.7-51.1) pre-treatment to a mean of $38.0 \mathrm{~cm} / \mathrm{s}$ (IQR 31.6-45.1) at one-month post-treatment. Although the average PSV at one-month follow-up increased to greater than the defined lower limit of normal $(35 \mathrm{~cm} / \mathrm{s})$, these changes were not statistically significant $(\mathrm{p}=$ 0.986) [14].

On univariate analysis, smoking status appeared to be associated with increased likelihood of treatment failure (OR 6.90, $\mathrm{p}=0.074$ ), but this failed to reach statistical significance. No other pretreatment characteristics appeared to be associated with success of treatment $(\mathrm{p}>0.1)$.

Minor adverse events involving 1.3\% $(n=2)$ of 150 treatment sessions necessitated altering shock delivery due to mild discomfort, but no sessions were terminated. Importantly, no adverse effects including penile hematoma, corporal rupture, or deep vein thrombosis were noted.

\section{Discussion}

Shockwave therapy in the form of lithotripsy has long been used in the treatment of urolithiasis in order to directly break up stones at the time of treatment. However, shockwave therapy in the treatment of ED relies on delayed outcomes from tissue response to the damage created by shockwaves [15]. The hypothesized response mechanism is centered on local neovascularization. Wang et al. demonstrated substantially increased levels of endothelial nitric oxide synthase, vascular endothelial growth factor, and proliferating cell nuclear antigen when shockwave therapy was applied to Achilles tendons of rabbits [16]. Nerve regeneration and mesenchymal stem cell migration/proliferation has also been suggested as an outcome of 
shockwave therapy [15]. While these molecular-level changes have been demonstrated in vitro and in animal studies, the complete mechanism has not been fully understood in humans and research is ongoing.

Shockwave therapy has been employed in several other areas of medicine with promising results. After numerous porcine studies revealed LISWT improved regional myocardial blood flow and improved left ventricular remodeling after reperfusion injury, it was investigated in humans with ischemic heart disease [17-19]. In a small study by Kikuchi et al., promising results were reported in functional outcomes such as improved chest pain symptom scores and reduced nitroglycerin use, as well as objective outcomes like ejection fraction and stroke volume when cardiac LISWT was administered to men with severe ischemic disease [20]. A meta-analysis published in 2017 inclusive of 39 studies revealed consistent improvements in various clinical metrics such as quality of life and myocardial perfusion indices with the use of targeted LISWT in patients with stable coronary artery disease [21]. Shockwave therapy has been investigated in the treatment of diabetic foot ulcers with promising results when compared to hyperbaric oxygen therapy [22]. It has also been reported on extensively in the orthopedic literature, mainly in the treatment of over-use tendinopathies with success rates ranging from 65-91\%. Shockwave therapy has even been FDA approved for the treatment of plantar fasciitis and lateral epicondylitis [5].

Several meta-analyses published within the past 10 years have found that LISWT can significantly improve patients' symptoms as evaluated by validated questionnaires [7-9]. Lu et al. included 14 studies evaluating the efficacy of LISWT in patients with ED and reported statistically significant improvements in IIEF scores (95\% CI, 0.99-3.00; p 0.0001) and Erection Hardness Scores (EHS) (95\% CI, 0.04-0.29; p = 0.01) [7]. A 2017 meta-analysis inclusive of 602 patients with ED who underwent treatment with either sham or LISWT demonstrated that LISWT had an average IIEF score increase of 6.40 (95\% CI 1.78-11.02; P < 0.0001) compared to 1.65 (95\% CI 0.92-2.39; P < 0.0001) compared to sham [8]. Vardi et al. reported improvements in IIEF scores from a mean of 13.5 at baseline to 20.9 one month after LISWT in 20 men with vasculogenic ED, congruent with the significant improvements seen in our IIEF scores from a baseline of 13 to 18 at onemonth post-treatment [11]. Published data on LIWST is promising, but young and therefore the AUA considers it investigational. However, the European Association of Urology includes LIWST as a treatment option for patients with vasculogenic ED [23]. Our data supports the efficacy of LISWT as a treatment for mild to moderate ED, with $68 \%$ of patients experiencing improvement in symptoms and an average improvement in cavernosal artery PSV of approximately $4 \mathrm{~cm} / \mathrm{s}$ at one-month follow-up.

The duration of treatment efficacy remains unclear. A meta-analysis from Man and Li evaluated 637 patients who received LISWT from years 2005-2017 for ED [9]. The authors utilized significant improvements in IIEF scores and EHS as their markers of treatment success; through this definition, therapeutic efficacy was noted up to three months post-treatment. Although our results do not extend past one-month follow-up, given the latter, perhaps peak improvements in erectile function were missed due to lack of longer-term data.

Shockwave devices vary in manufacturer, energy capacity, and shockwave delivery. Shockwaves may be delivered in a pinpoint manner, linear manner, or in a linear tissue-coverage manner. The device utilized in our study delivered shocks in a linear, tissue-coverage fashion. Our goal in utilizing a tissue-coverage approach was to deliver a more homogenous dose of energy with the hope that uniform microvascular changes would occur within the corpora bilaterally. In a study of 58 patients with mild to severe ED, Reisman et al. utilized a similar linear-tissue coverage device but with a curved applicator that partially wrapped around each corpora for maximal engagement with $81 \%$ reported success rate [12]. In a small study published in 2013, the use of a pinpoint shock applicator resulted in statistically significant improvements in IIEF scores and penile blood flow at one-month follow-up [24]. Despite promising data on individual shockwave applicators, there is limited data on device comparison and superiority.

It is rare to find consistent treatment protocols across trials utilizing LISWT for ED. Vardi et al. performed bi-weekly sessions for three weeks, repeated after a three-week treatment holiday [11]. They also required a four-week PDE-5I washout period prior to starting shockwave therapy. A study comparing efficacy of 6 versus 12 treatment sessions within a six-week period reported improved sexual performance in those undergoing 12 sessions [25]. Shock and energy delivery are also variable. In most studies, shocks delivered range from 600 to 5,000 with energy intensities of 0.009 to $0.16 \mathrm{~mJ} / \mathrm{mm}[11-12,24]$. Kalyvianakis et al.'s data suggests improved subjective and objective outcomes when utilizing $0.10 \mathrm{~mJ} / \mathrm{mm}^{2}$ versus $0.05 \mathrm{~mJ} / \mathrm{mm}^{2}$, but no difference in two versus three weekly treatment sessions for a total of 12 sessions [25]. A 2017 metaanalysis suggests that lower energy density $\left(0.09 \mathrm{~mJ} / \mathrm{mm}^{2}, 95 \% \mathrm{CI}, 0.87-7.42 ; \mathrm{P}=.01\right)$, increased number of pulses (3000 pulses per treatment, $95 \% \mathrm{CI}, 3.18-7.05, \mathrm{P}<.0001)$, and a shorter total treatment course $(<6$ weeks, 95\% CI, 0.54-6.93; $\mathrm{P}=.02$ ) resulted in better therapeutic efficacy [9]. Contrastingly, Lu et al. found that variation in machine design, application technique and protocol did not impact their outcomes [7]. Ultimately, the variances in protocols and shockwave devices make comparison and standardized clinical application of this therapy a challenge.

In a 2013 meta-analysis, Cao et al. reported a significantly increased risk of ED in patients that were either former or current smokers with odds ratios of 1.29 (95\% CI: 1.07 to 1.47 ) and 1.51 (95\% CI: 1.34 to 1.71 ), respectively [26]. Smoking has been shown to decrease the bioavailability of nitric oxide leading to 
endothelial dysfunction by the proposed mechanism of free radical formation and aromatic compound accumulation [27]. Smoking status negatively impacted the success rates of LISWT defined as an increase of 2 and 5 points on IIEF scores for mild and moderate ED, respectively, in a study that included 15 men with mild to moderate vasculogenic ED [28]. In this study, patients with a smoking index of greater than 20 had a statistically significant higher likelihood of treatment failure compared to those with a smoking index of less than 20 (50\% vs. 9\%) [28]. Our data is supportive, also revealing that smoking is associated with treatment failure.

LISWT has been reported to be a safe treatment modality for patients with ED. In a meta-analysis published in 2019 that reviewed eight randomized controlled clinical trials investigating LISWT for ED, no adverse events were reported across all studies [29]. Some concern may be raised for increased risk of bleeding when treating patients on anticoagulation. A study by Kalyvianakis et al. that included 138 men on anticoagulation or antiplatelet therapy who underwent LISWT for ED reported no bleeding events or other adverse reactions [30]. In our cohort, anticoagulation/antiplatelet use was not elucidated, but the only adverse event reported was discomfort during treatment for two patients, which resolved with device repositioning and did not require termination of either session.

There are several limitations to our study, including the small cohort with limited demographics, short-term follow-up, and lack of a control arm. Even with short-term follow-up of only one month, three patients were lost which could significantly impact results in this small cohort. Although protocol was standardized, variability in ultrasound technician may impact patient outcomes and results. Additionally, duplex ultrasounds were individually reviewed by radiologists and there is likely inherent variation in PSV results and interpretation.

\section{Conclusions}

LISWT may be a reliable treatment option in patients experiencing mild to moderate ED. The results of this study demonstrate short-term efficacy with overall improvement in subjective outcomes measured by IIEF and EDITS validated questionnaires as well as objectively with improvements seen in PSV of the cavernosal arteries. Data is limited due to lack of long-term results and small sample size. Future studies with larger cohorts and standardized protocols are needed to better delineate the long-term efficacy and feasibility of LISWT as a recommended treatment option for patients with mild to moderate ED.

\section{Additional Information \\ Disclosures}

Human subjects: Consent was obtained by all participants in this study. Western IRB issued approval 20172573. Animal subjects: All authors have confirmed that this study did not involve animal subjects or tissue. Conflicts of interest: In compliance with the ICMJE uniform disclosure form, all authors declare the following: Payment/services info: All authors have declared that no financial support was received from any organization for the submitted work. Financial relationships: All authors have declared that they have no financial relationships at present or within the previous three years with any organizations that might have an interest in the submitted work. Other relationships: All authors have declared that there are no other relationships or activities that could appear to have influenced the submitted work.

\section{References}

1. Johannes CB: Incidence of erectile dysfunction in men 40 to 69 years old: longitudinal results from the Massachusetts male aging study. J Urol. 2000, 163:460-463. 10.1016/S0022-5347(05)67900-1

2. Miner M, Nehra A, Jackson G, et al.: All men with vasculogenic erectile dysfunction require a cardiovascular workup. Am J Med. 2014, 127:174-182. 10.1016/j.amjmed.2013.10.013

3. Maiorino M, Bellastella G, Esposito K: Lifestyle modifications and erectile dysfunction: what can be expected?. Asian J Androl. 2015, 17:5-10. 10.4103\%2F1008-682X.137687

4. Musa Z, El-Assmy A, Shokry AM, Shokeir AA, Zween T, Al-Kenawy MR: Long-term effectiveness and predictors of success of low-intensity shockwave therapy in phosphodiesterase type 5 inhibitors nonresponders. Arab J Urol. 2020, 18:54-58. 10.1080/2090598X.2019.1688072

5. Wang CJ: Extracorporeal shockwave therapy in musculoskeletal disorders . J Orthop Surg Res. 2012, 7:11. 10.1186/1749-799X-7-11

6. Chung E, Wang J: A state-of-art review of low intensity extracorporeal shock wave therapy and lithotripter machines for the treatment of erectile dysfunction. Expert Rev Med Devices. 2017, 14:929-934. 10.1080/17434440.2017.1403897

7. Lu Z, Lin G, Reed-Maldonado A, Wang C, Lee YC, Lue TF: Low-intensity extracorporeal shock wave treatment improves erectile function: a systematic review and meta-analysis. Eur Urol. 2017, 71:223-233. 10.1016/j.eururo.2016.05.050

8. Clavijo R, Kohn T, Kohn J, Ramasamy R: Effects of low-intensity extracorporeal shockwave therapy on erectile dysfunction: a systematic review and meta-analysis. J Sex Med. 2017, 14:27-35. 10.1016/j.jsxm.2016.11.001

9. Man L, Li G: Low-intensity extracorporeal shock wave therapy for erectile dysfunction: a systematic review and meta-analysis. Urology. 2018, 119:97-103. 10.1016/j.urology.2017.09.011

10. Cappelleri J, Rosen R, Smith M, Mishra A, Osterloh IH: Diagnostic evaluation of the erectile function domain 
of the international index of erectile function. Urology. 1999, 54:346-351. 10.1016/S0090-4295(99)00099-0

11. Vardi Y, Appel B, Jacob G, Massarwi O, Gruenwald I: Can low-intensity extracorporeal shockwave therapy improve erectile function? A 6-month follow-up pilot study in patients with organic erectile dysfunction. Eur Urol. 2010, 58:243-248. 10.1016/j.eururo.2010.04.004

12. Reisman Y, Hind A, Varaneckas A, Motil I: Initial experience with linear focused shockwave treatment for erectile dysfunction: a 6-month follow-up pilot study. Int J Impot Res. 2015, 27:108-112. 10.1038/ijir.2014.41

13. Cappelleri J, Tseng L, Stecher V, Althof SE: Clinically important difference on the Erectile Dysfunction Inventory of Treatment Satisfaction questionnaire in patients with erectile dysfunction. Int J Clin Pract. 2018, 72:13073. 10.1111/ijcp.13073

14. Wein A: Physiology of penile erection and pathophysiology of erectile dysfunction. Campbell-Walsh Urology. Elsevier, 2015. 612.

15. Vardi Y, Appel B, Kilchevsky A, Gruenwald I: Does low intensity extracorporeal shock wave therapy have a physiological effect on erectile function? Short-term results of a randomized, double-blind, sham controlled study. J Urol. 2012, 187:1769-1775. 10.1016/j.juro.2011.12.117

16. Wang C, Wang FS, Yang KD, Weng LH, Hsu CC, Huang CS, Yang LC: Shock wave therapy induces neovascularization at the tendon-bone junction. A study in rabbits. J Orthop Res. 2003, 21:984-989. 10.1016/S0736-0266(03)00104-9

17. Nishida T, Shimokawa H, Oi K, et al.: Extracorporeal cardiac shock wave therapy markedly ameliorates ischemia-induced myocardial dysfunction in pigs in vivo. Circulation. 2004, 110:3055-3061. 10.1161/01.CIR.0000148849.51177.97

18. Ito Y, Ito K, Shiroto T, et al.: Cardiac shock wave therapy ameliorates left ventricular remodeling after myocardial ischemia-reperfusion injury in pigs in vivo. Coron Artery Dis. 2010, 21:304-311. 10.1097/MCA.0b013e32833aec62

19. Uwatoku T, Ito K, Abe K, Oi K, Hizume T, Sunagawa K, Shimokawa H: Extracorporeal cardiac shock wave therapy improves left ventricular remodeling after acute myocardial infarction in pigs. Coron Artery Dis. 2007, 18:397-404. 10.1097/MCA.0b013e328089f19b

20. Kikuchi Y, Ito K, Ito Y, et al.: Double-blind and placebo-controlled study of the effectiveness and safety of extracorporeal cardiac shock wave therapy for severe angina pectoris. Circ J. 2010, 74:589-591. 10.1253/circj.CJ-09-1028

21. Burneikaitė G, Shkolnik E, Čelutkienè J, et al.: Cardiac shock-wave therapy in the treatment of coronary artery disease: systematic review and meta-analysis. Cardiovasc Ultrasound. 2017, 15:11. 10.1186/s12947017-0102-y

22. Hitchman L, Totty J, Raza A, et al.: Extracorporeal shockwave therapy for diabetic foot ulcers: a systematic review and meta-analysis. Ann Vasc Surg. 2019, 56:330-339. 10.1016/j.avsg.2018.10.013

23. Montague DK, Jarow JP, Broderick GA, et al.: The management of erectile dysfunction: an AUA update . I Urol. 2005, 174:230-239. 10.1097/01.ju.0000164463.19239.19

24. Gruenwald I, Appel B, Kitrey N, Vardi Y: Shockwave treatment of erectile dysfunction. Ther Adv Urol. 2013, 5:95-99. 10.1177\%2F1756287212470696

25. Kalyvianakis D, Memmos E, Mykoniatis I, Kapoteli P, Memmos D, Hatzichristou D: Low-intensity shockwave therapy for erectile dysfunction: a randomized clinical trial comparing 2 treatment protocols and the impact of repeating treatment. J Sex Med. 2018, 15:334-345. 10.1016/j.jsxm.2018.01.003

26. Cao S, Yin X, Wang Y, Zhou H, Song F, Lu Z: Smoking and risk of erectile dysfunction: systematic review of observational studies with meta-analysis. PLoS ONE. 2013, 8:e60443. 10.1371/journal.pone.0060443

27. McVary K, Carrier S, Wessells H: Smoking and erectile dysfunction: evidence based analysis . J Urol. 2001, 166:1624-1632. 10.1016/S0022-5347(05)65641-8

28. Pelayo-Nieto M, Linden-Castro E, Alias-Melgar A, Espinosa-Pérez Grovas D, Carreño-de la Rosa F, BertrandNoriega F, Cortez-Betancourt R: Linear shock wave therapy in the treatment of erectile dysfunction . Actas Urol Esp. 2015, 39:456-459. 10.1016/j.acuroe.2015.06.009

29. Mo D, Zhan XX, Cai HC, Shi HW, Meng J, Zhao J, Shang XJ: Efficacy and safety of low-intensity extracorporeal shock wave therapy in the treatment of ED: a meta-analysis of randomized controlled trials. Nat J Andr. 2019, 25:257-264.

30. Kalyvianakis D, Memmos D, Mykoniatis I, Kapoteli P, Hatzichristou D: Low-intensity shockwave therapy for erectile dysfunction: is it safe for patients on anticoagulant medication?. J Sex Med. 2019, 16:1478-1480. 10.1016/j.jsxm.2019.05.008 